Supporting Information

\title{
Ultralow-Ru Induced Bimetal Electrocatalysts with a Ru-Enriched and Mixed-Valence Surface Anchored on Hollow Carbon Matrix for Oxygen Reduction and Water Splitting
}

Guoning Li $\dagger, \perp$, Kaitian Zheng $\dagger, \perp$, Weisong Li $\uparrow$, Yongchao He $\dagger$ and Chunjian $\mathrm{Xu}^{*}, \dagger^{\dagger}$

School of Chemical Engineering and Technology, State Key Laboratory of Chemical

Engineering, Chemical Engineering Research Center, Tianjin University, Tianjin 300072 , China.

${ }^{\sharp}$ School of Chemical Engineering and Technology, China University of Mining \& Technology, Xuzhou 221116, China.

${ }^{\perp}$ These authors contributed equally to this work.

*Corresponding author. E-mail: cjxu@tju.edu.cn. 


\section{- EXPERIMENTAL SECTION}

\section{Material Characterization}

The scanning electron microscope (SEM, Hitachi S-4800) and transmission electron microscope (TEM, JEOL JEM-2100) were used to investigate the morphology of these samples. The XRD patterns were recorded by a D8 Advance Bruker X-ray diffractometer (XRD) at a scan rate of $5^{\circ} \mathrm{min}^{-1}$. Raman spectra were obtained using a Renishaw InVia Spectrometer with a $532 \mathrm{~nm}$ excitation laser. Nitrogen adsorption/desorption was performed on Auto-Sorb-iQA 3200-4 (Quantatech Co., USA). X-ray photoelectron spectroscopy (XPS) analysis was performed on a Thermo Scientific Spectrometer with an Escalab 250 Xi X-ray. Metal concentration was determined by Perkin Elmer Optima 5300 DV optic emission spectrometer (ICP). Electron paramagnetic resonance (EPR) measurements were performed on a Bruker model A300 spectrometer.

\section{Electrochemical Characterization}

The electrochemical tests were carried out by using a CHI 660E workstation and RDE equipment (ALS/ DY2323 Bi-potentiostat) with a conventional three-electrode system. A glassy carbon electrode (GCE, $3 \mathrm{~mm}$ of disk diameter), a platinum wire for ORR (or a graphite rod for $\mathrm{OER}$ and $\mathrm{HER}$ ), and $\mathrm{Ag} / \mathrm{AgCl}$ with saturated $\mathrm{KCl}$ were served as the working, counter and reference electrodes, respectively. $3 \mathrm{mg}$ of catalyst was dispersed in $1 \mathrm{ml}$ of mixture containing Nafion solution ( $4 \% \mathrm{v} / \mathrm{v})$ and isopropanol $(41 \% \mathrm{v} / \mathrm{v})$, water $(55 \%$ $\mathrm{v} / \mathrm{v}$ ) and then ultrasonicated for $1 \mathrm{~h}$ to form a catalyst ink. The mass loading of the prepared material on the GCE was $0.24 \mathrm{mg} \cdot \mathrm{cm}^{-2}$ and that of reference substance $(\mathrm{Pt} / \mathrm{C}$ for ORR/HER and $\mathrm{RuO}_{2}$ for $\mathrm{OER}$ ) was $0.13 \mathrm{mg} \mathrm{cm}^{-2}$. High purity oxygen or nitrogen was bubbled into the electrolyte for at least $0.5 \mathrm{~h}$ before the tests. The $\mathrm{CV}$ measurements were carried out in 
$\mathrm{N}_{2}$ or $\mathrm{O}_{2}$-saturated electrolyte with a scan rate of $50 \mathrm{mV} \mathrm{s}^{-1}$. The linear sweep voltammogram (LSV) tests were conducted on a rotating disk electrode (RDE). All the LSV curves for OER and HER were IR-corrected unless otherwise specified. The rotating speed of RDE varied from 400 to $2250 \mathrm{rpm}$ with a scan rate of $10 \mathrm{mV} \mathrm{s}^{-1}$. The electron transfer number (n) for ORR was calculated by the Koutecky-Levich equations:

$$
\begin{aligned}
& \frac{1}{J}=\frac{1}{J_{L}}+\frac{1}{J_{K}}=\frac{1}{B \omega^{1 / 2}}+\frac{1}{J_{K}} \\
& B=0.62 n F C_{0} D_{0}^{2 / 3} v^{-1 / 6}
\end{aligned}
$$

where $\mathrm{J}$ represents the measured current density. $\mathrm{J}_{\mathrm{L}}$ and $\mathrm{J}_{\mathrm{K}}$ are the kinetic current density and limiting diffusion current density, respectively. $\omega$ is the rotation rate of RDE test. F is the Faraday constant of $96485 \mathrm{C} \mathrm{mol}^{-1}, \mathrm{C}_{0}$ is the bulk concentration of $\mathrm{O}_{2}$ dissolved $(1.2 \times$ $\left.10^{-3} \mathrm{~mol} \mathrm{~L}^{-1}\right), \mathrm{D}_{0}$ is the diffusion coefficient of $\mathrm{O}_{2}\left(1.9 \times 10^{-5} \mathrm{~cm}^{2} \mathrm{~s}^{-1}\right)$, and $v$ is the kinetic viscosity of $0.1 \mathrm{M} \mathrm{KOH}\left(0.01 \mathrm{~cm}^{2} \mathrm{~s}^{-1}\right)$.

For overall water splitting test, two pieces of CoRu-O/A@HNC-2 supported on carbon cloths $\left(1 \times 1 \mathrm{~cm}^{2}\right)$ are used as both the anode and the cathode in a two-electrode system in 1 $\mathrm{M} \mathrm{KOH}$. The mass loading of the catalyst in carbon cloth is about $0.27 \mathrm{mg} / \mathrm{cm}^{2}$. 


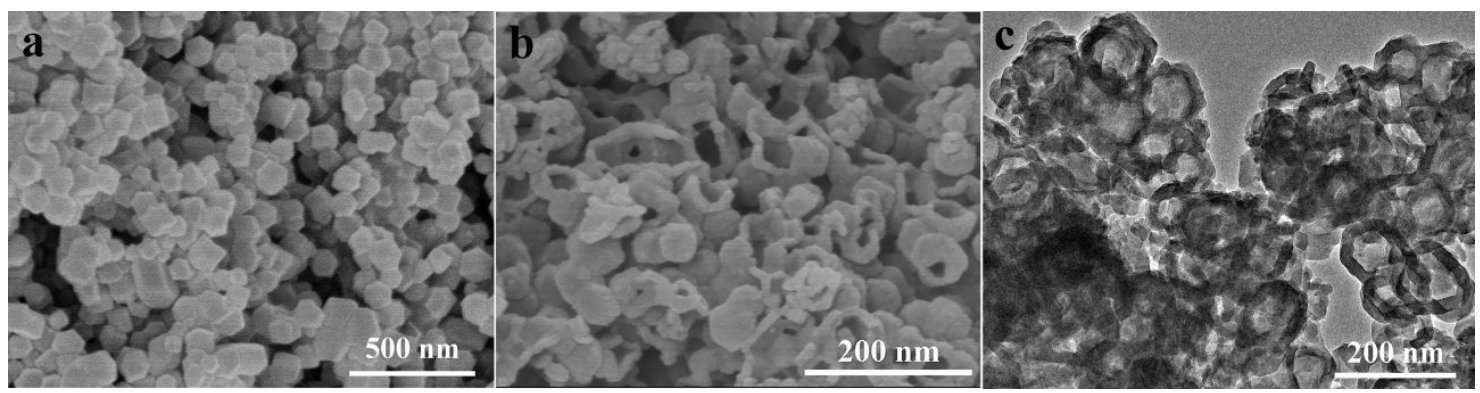

Figure S1. SEM images of (a) Zn/Co-ZIF and (b) RuHZIF. (c) TEM images of RuHZIF.

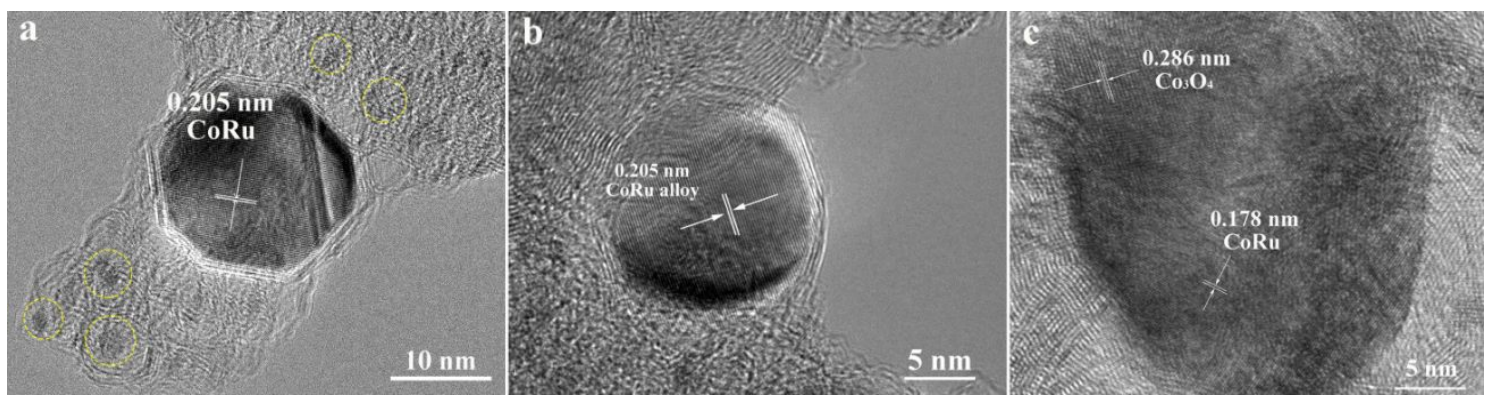

Figure S2. HRTEM images of (a) CoRu-A@HNC, (b) and (c) CoRu-O/A@HNC-2.

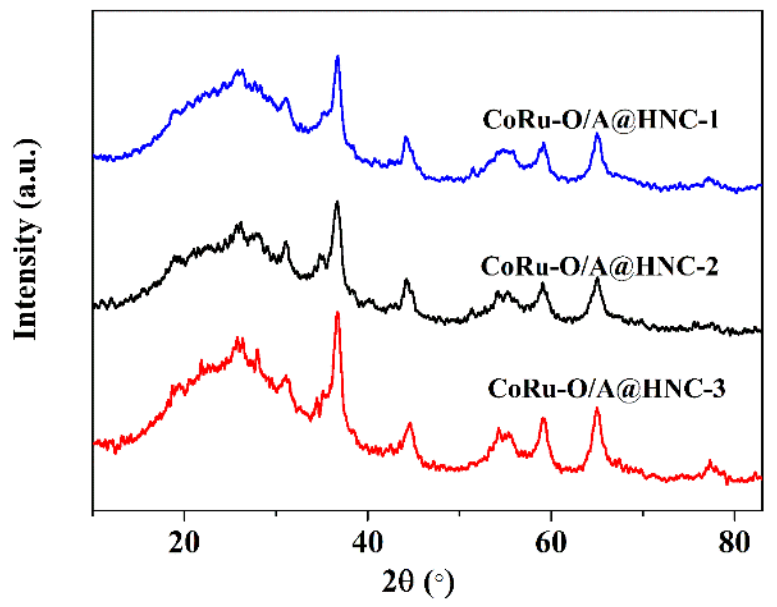

Figure S3. XRD patterns of different CoRu-O/A@HNC-T. 


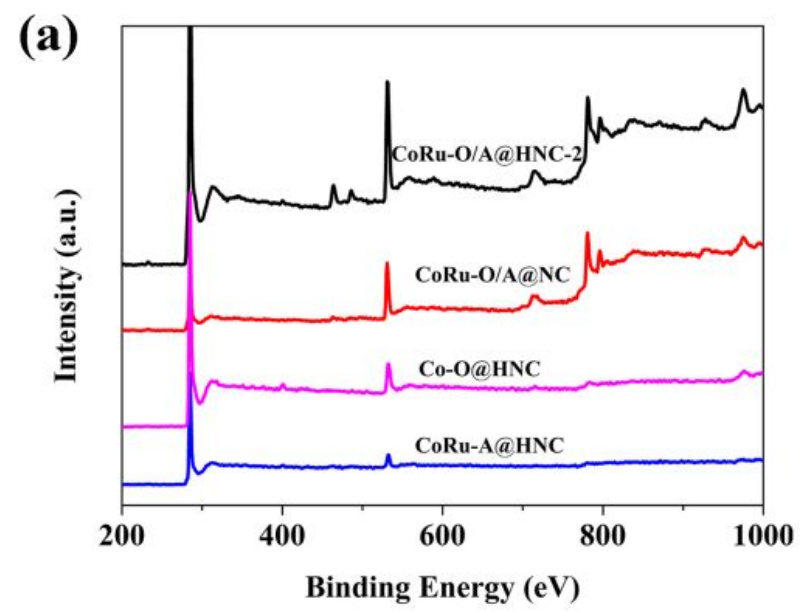

Figure S4. (a) The survey XPS spectra of various catalysts.
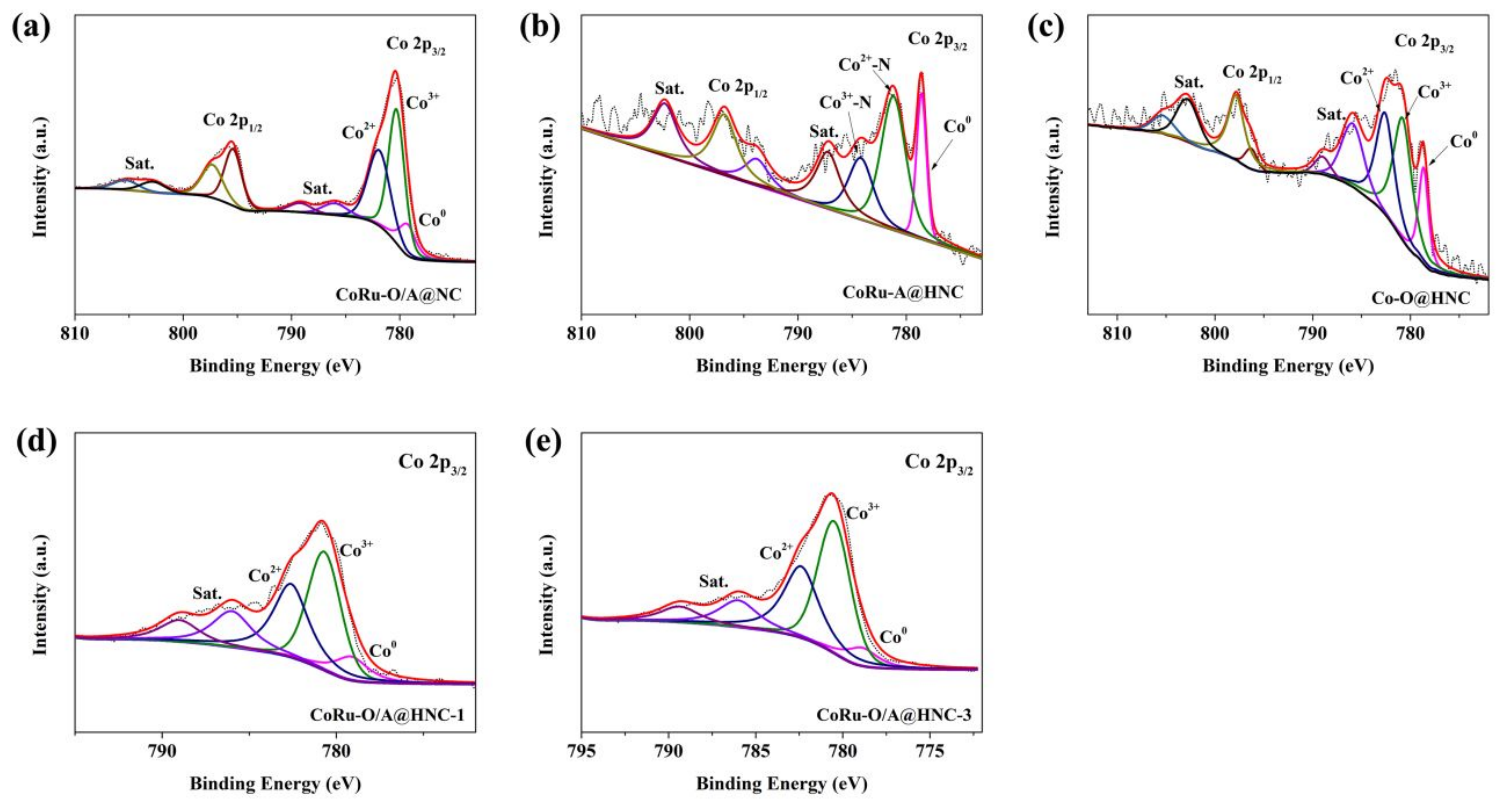

Figure S5. Co 2p high-resolution XPS spectra of (a) CoRu-O/A@NC, (b) CoRu-A@HNC, (c) CoO@HNC, (d)CoRuO/A@HNC-1, (e)CoRuO/A@HNC-3. 

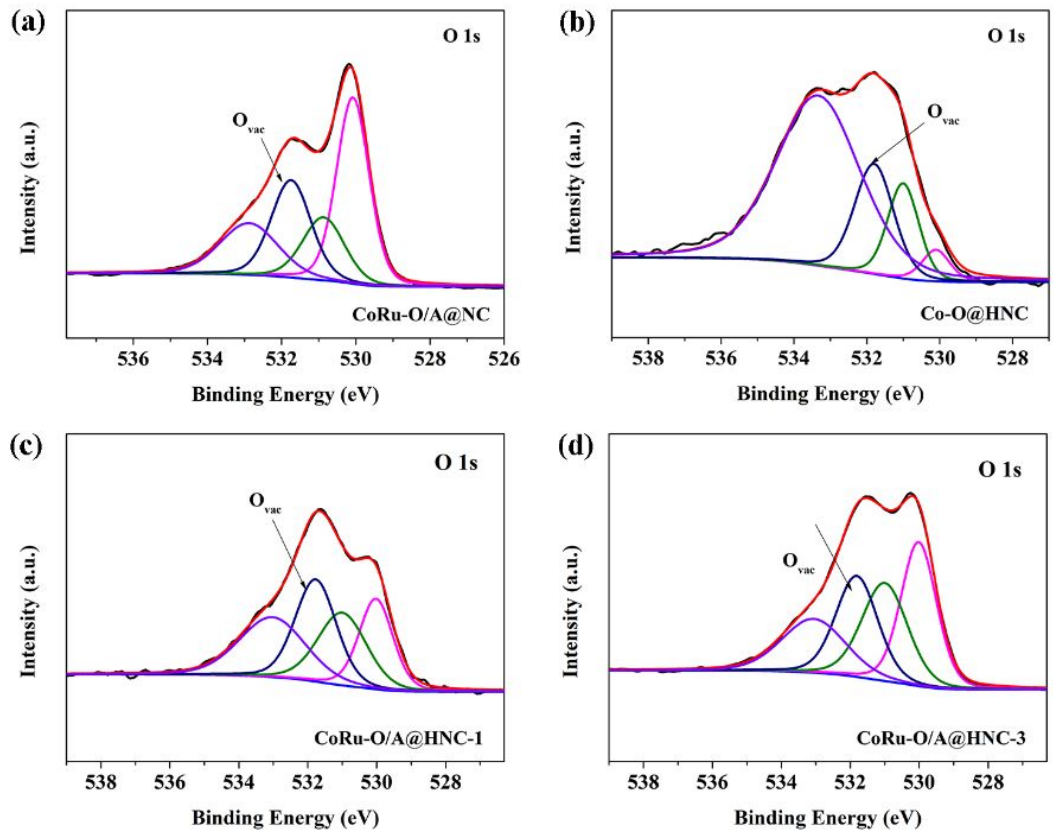

Figure S6. O 1s high-resolution XPS spectra of (a) CoRu-O/A@NC, (b) Co-O@HNC, (c) CoRuO/A@HNC-1, (d)CoRuO/A@HNC-3.
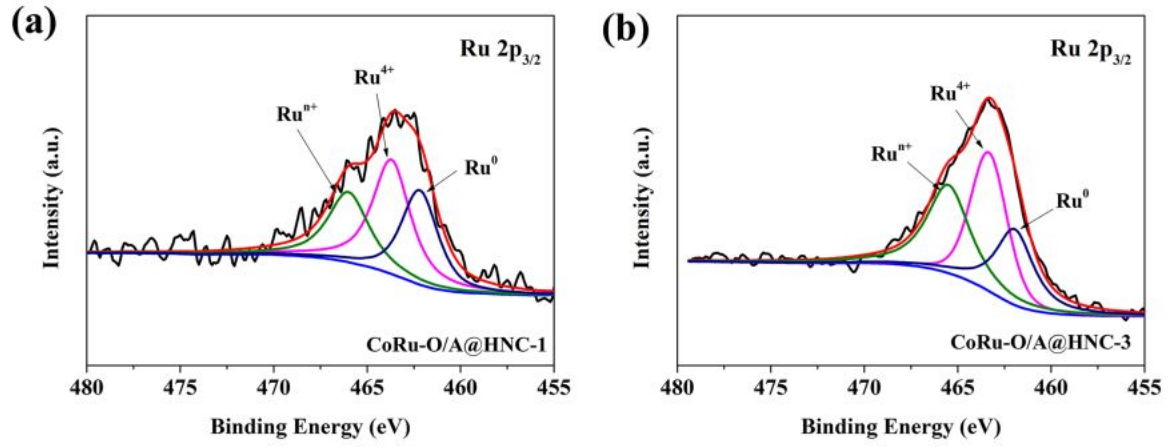

Figure S7. (a) $\mathrm{Ru} 2 \mathrm{p}_{3 / 2}$ high-resolution XPS spectra of (a) CoRu-O/A@HNC-1, (b) CoRuO/A@HNC-3. 

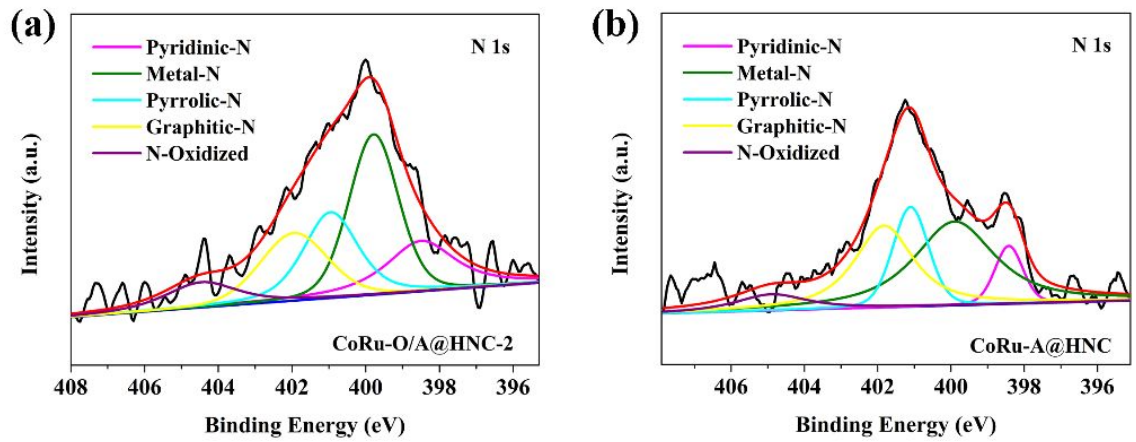

Figure S8. N 1s high-resolution XPS spectra of (a) CoRu-O/A@HNC-2 and (b) CoRu-A@HNC.
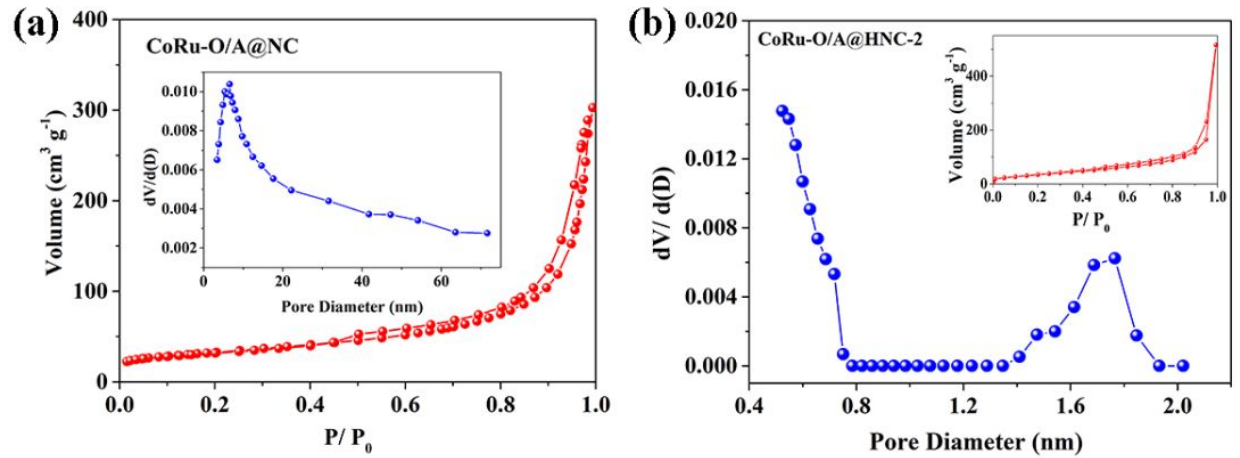

Figure S9. (a) $\mathrm{N}_{2}$ adsorption-desorption isotherm of CoRu-O/A@NC, (b) DFT pore size distribution for micropores of CoRu-O/A@HNC-2.

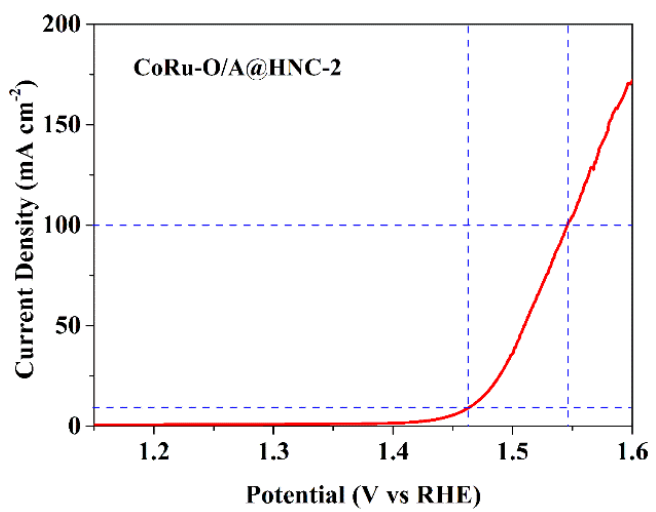

Figure S10. OER polarization curve of CoRu-O/A@HNC-2 on a GCE in $1 \mathrm{M} \mathrm{KOH}$. 

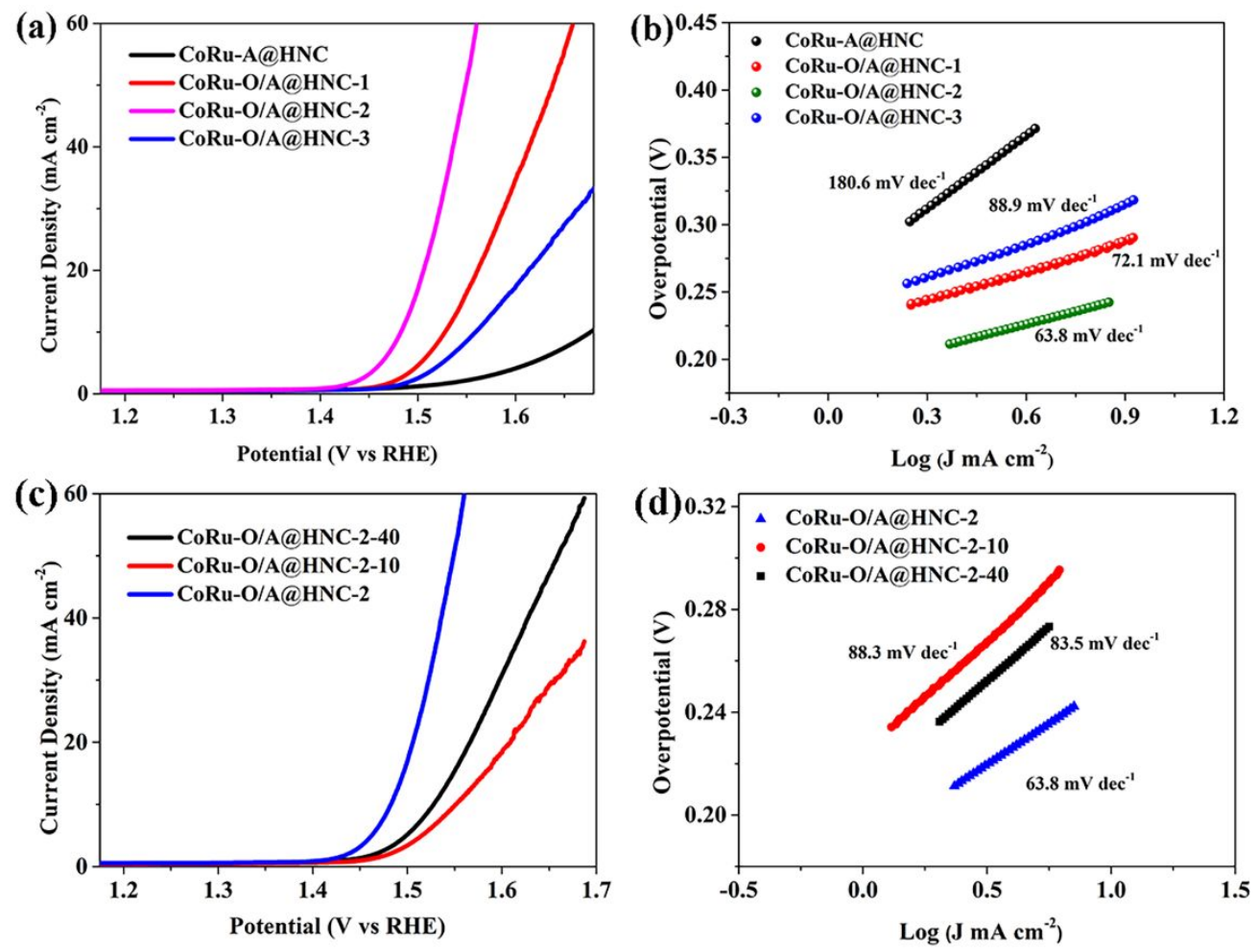

Figure S11. (a) OER polarization curves, (b) the corresponding Tafel slopes of the samples obtained at different oxidation time. (c) OER polarization curves, (d) the corresponding Tafel slopes of the samples obtained by different additive amount of $\mathrm{Ru}$ source.

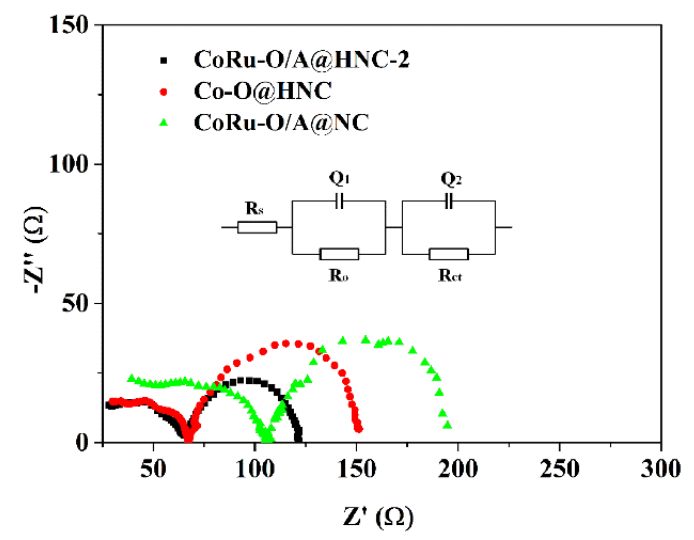

Figure S12. The Nyquist plots of CoRu-O/A@HNC-2, Co-O@HNC,CoRu-O/A@NC. 


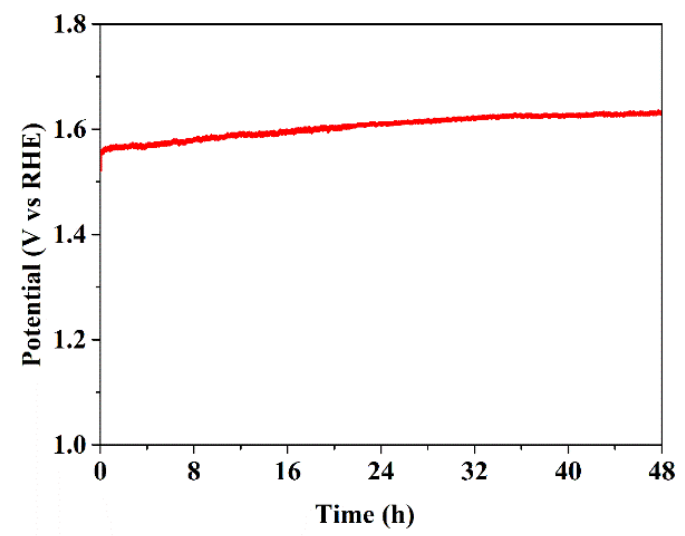

Figure S13. Chronopotentiometry tests of CoRu-O/A@HNC-2 for OER at $50 \mathrm{~mA} \mathrm{~cm}{ }^{-2}$.

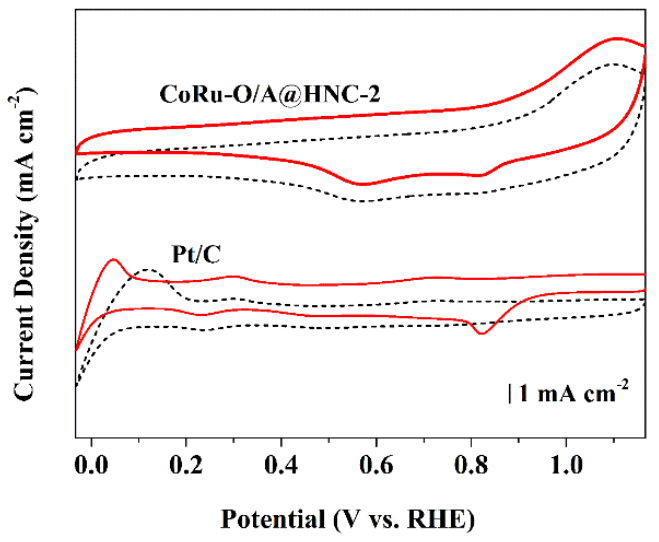

Figure S14. ORR CV curves of CoRu-O/A@HNC-2 and Pt/C.
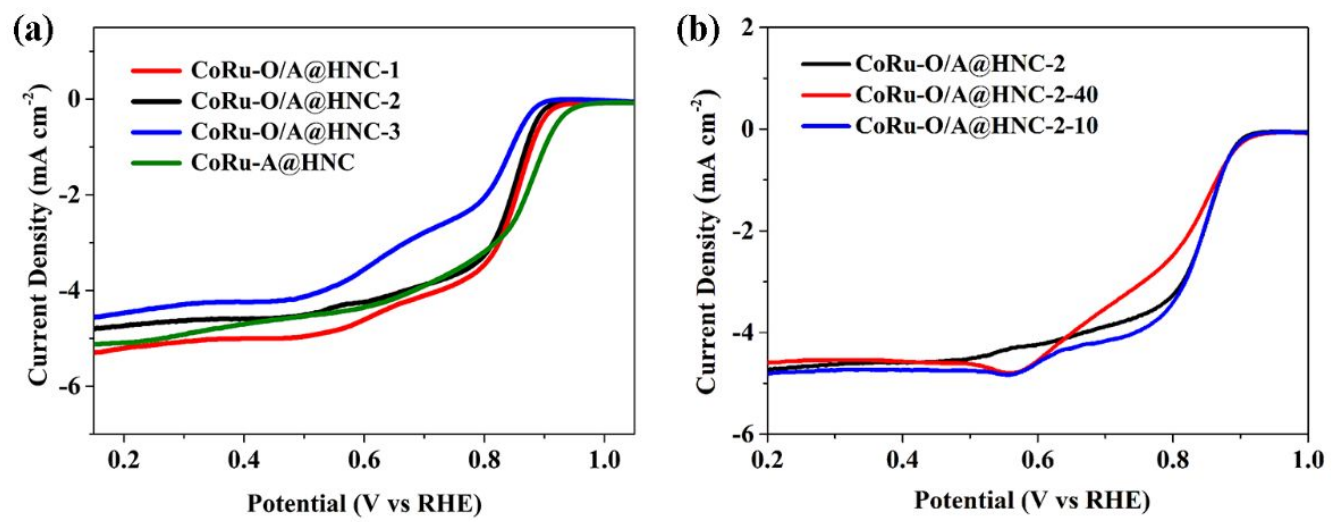

Figure S15. (a) ORR polarization curves of the CoRu-O/A@HNC-T catalysts and CoRu-A@HNC. (b) ORR polarization curves of the samples obtained by different additive amount of Ru source. all the tests were conducted in $0.1 \mathrm{M} \mathrm{KOH}$. 

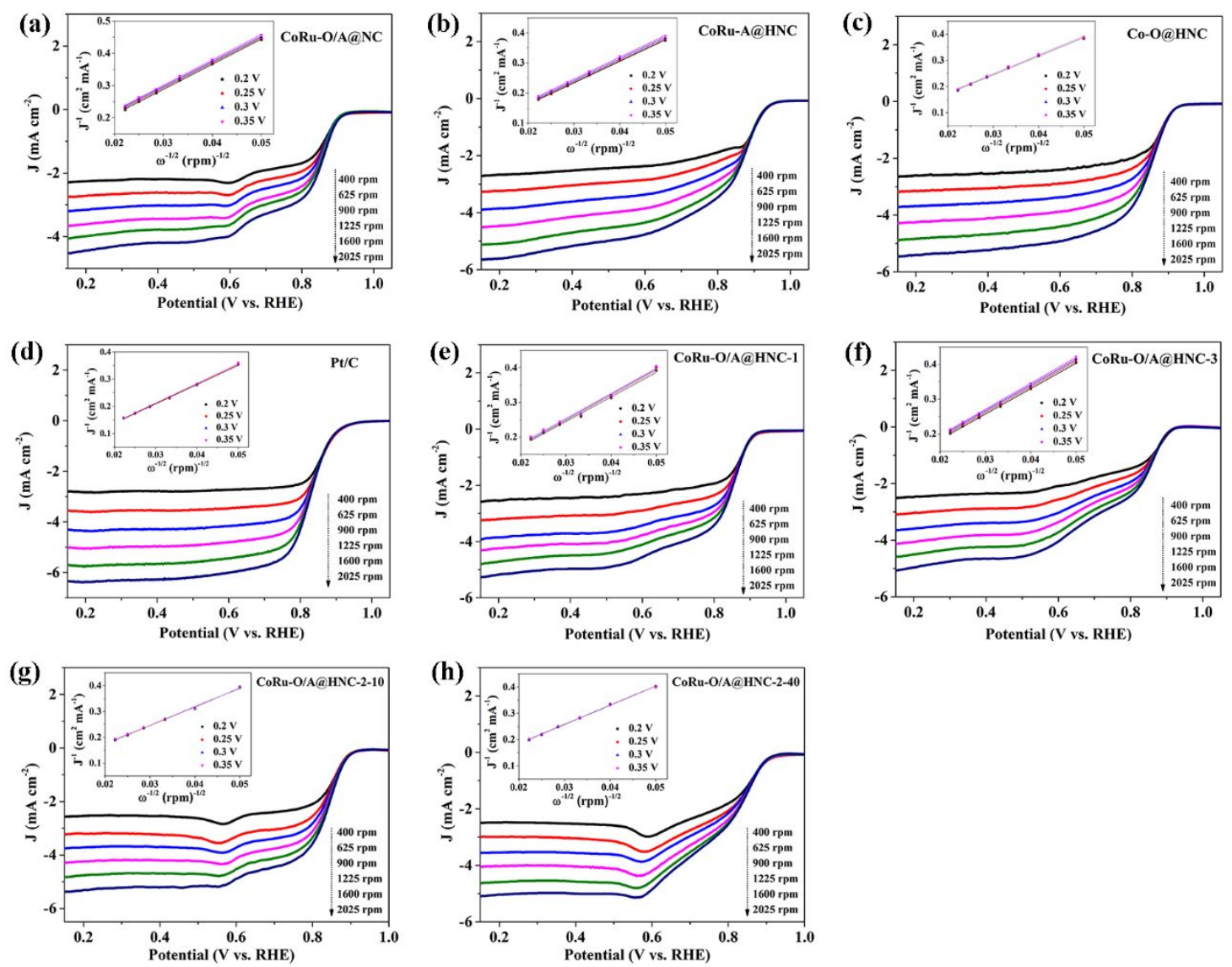

Figure S16. LSV curves of (a) CoRu-O/A@NC, (b) CoRu-A@HNC, (c) Co-O @HNC, (d) Pt/C, (e) CoRu-O/A@HNC-1, (f) CoRu-O/A@HNC-3, (g)CoRu-O/A@HNC-2-10, (h)CoRu-O/A@HNC-240 at different rotation speeds with the insets showing the $\mathrm{K}-\mathrm{L}$ plots at different potentials in $0.1 \mathrm{M}$ $\mathrm{KOH}$.

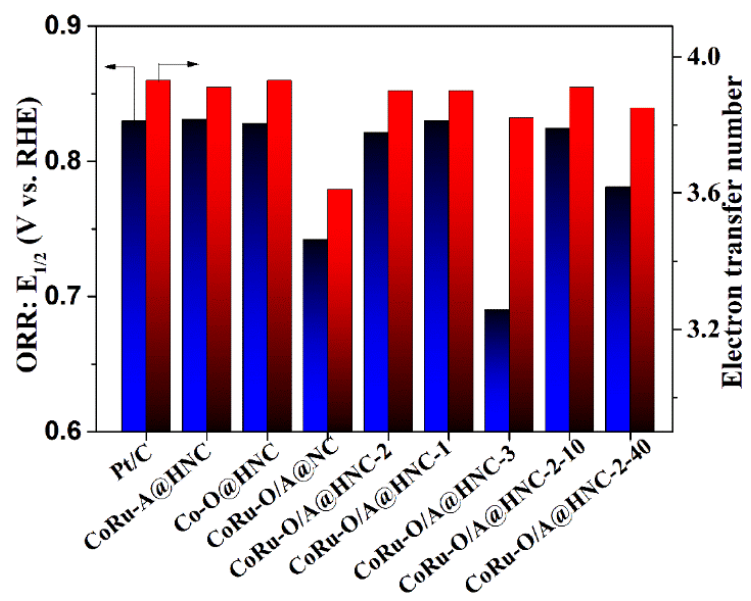

Figure S17. The summarized $\mathrm{E}_{1 / 2}$ and electron transfer numbers of various catalysts in $0.1 \mathrm{M} \mathrm{KOH}$. 

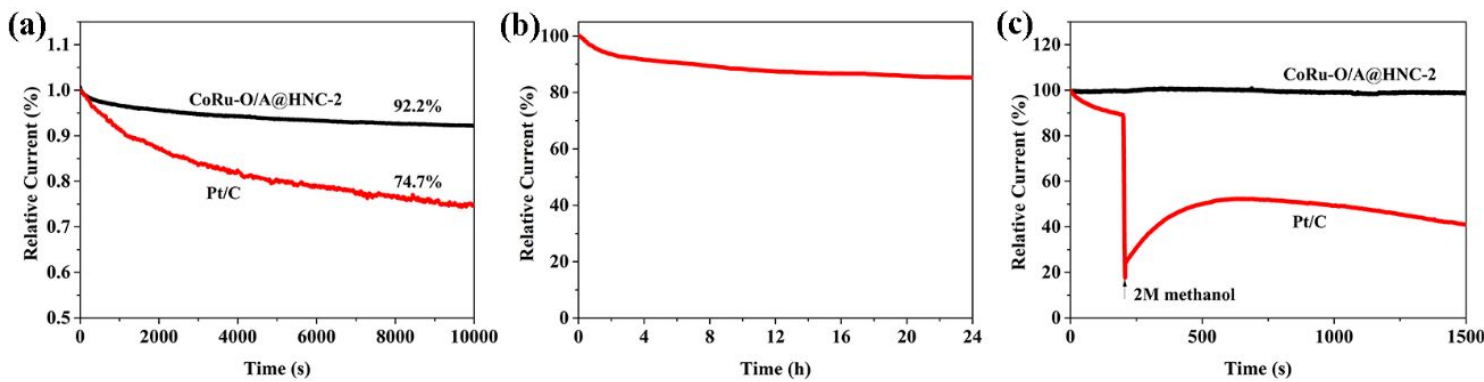

Figure S18. Chronoamperometric responses at $0.5 \mathrm{~V}$ vs RHE of (a) CoRu-O/A@HNC-2 and Pt/C for 10000s, (b) CoRu-O/A@HNC-2 for 24h. (c) Methanol tolerance test at $0.5 \mathrm{~V}$ vs RHE of CoRuO/A@HNC-2 and Pt/C.
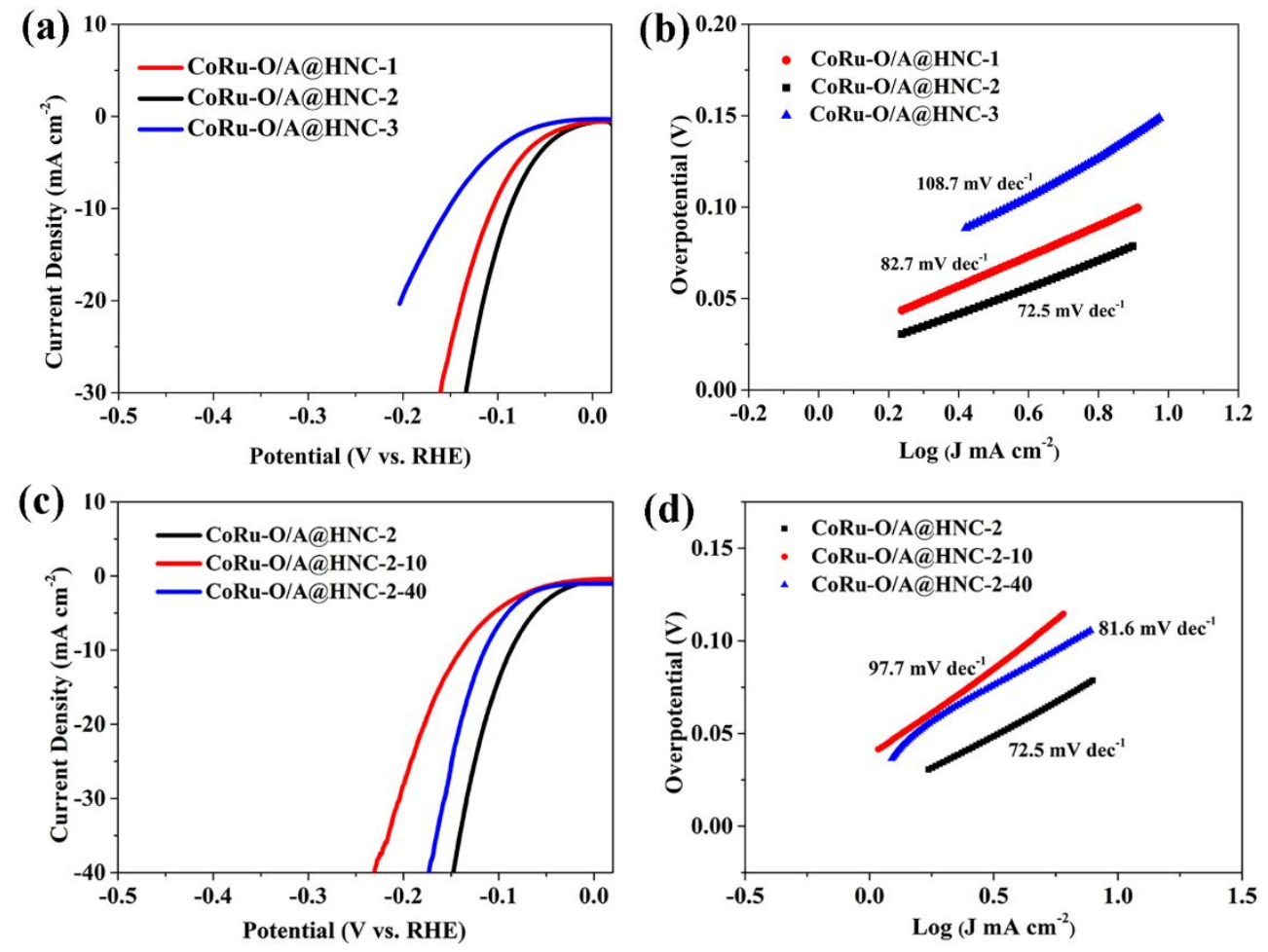

Figure S19. (a) HER polarization curves, (b) the corresponding Tafel slopes of the samples obtained at different oxidation time. (c) HER polarization curves, (d) the corresponding Tafel slopes of the samples obtained by different additive amount of Ru source. 


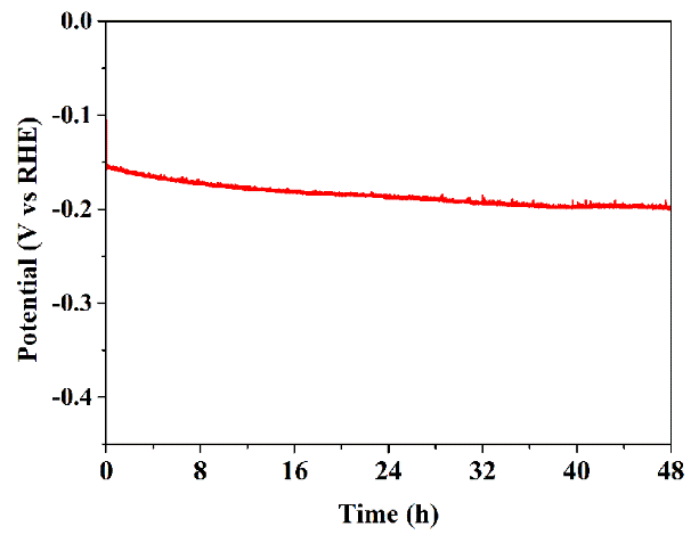

Figure S20. Chronopotentiometry tests of CoRu-O/A@HNC-2 for HER at $50 \mathrm{~mA} \mathrm{~cm}{ }^{-2}$.

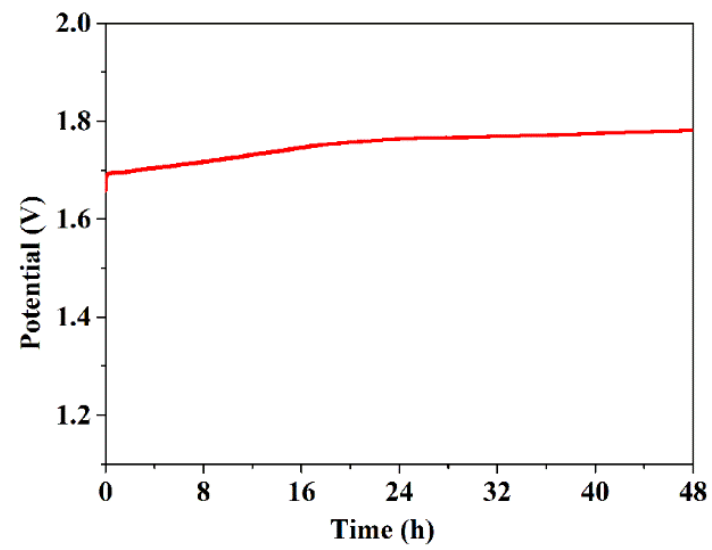

Figure S21. Chronopotentiometry tests of CoRu-O/A@HNC-2 for water splitting at $50 \mathrm{~mA} \mathrm{~cm}{ }^{-2}$.

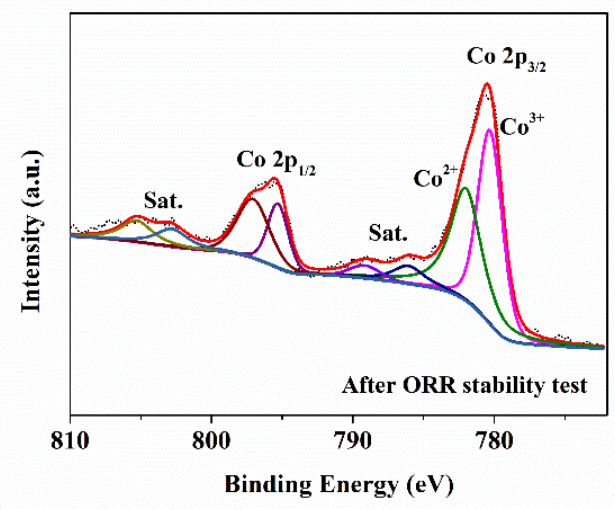

Figure S22. The Co 2p XPS spectrum of CoRu-O/A@HNC-2 after ORR stability test. 
Table S1. The content of Co and Ru, and Co/Ru molar ratio of CoRu-O/A@HNC-2 determined by ICP-OES and XPS

\begin{tabular}{ccc}
\hline & ICP-OES/ wt \% & XPS/ at\% \\
\hline Co & 25.47 & 3.68 \\
\hline Co & 1.28 & 1.44 \\
\hline Ru molar ratio & 34.1 & 2.6 \\
\hline
\end{tabular}

Table S2. Comparison of other recently reported transition metal-based OER electrocatalysts in alkaline conditions.

\begin{tabular}{|c|c|c|c|}
\hline Catalysts & Electrolyte & $E_{\mathrm{j}=10}(\mathrm{~V}$ vs. $\mathrm{RHE})$ & References \\
\hline \multirow[t]{3}{*}{ CoRu-O/A@HNC-2 } & 1 М КОН & 1.464 & \\
\hline & & & This work \\
\hline & 0.1 М КОН & 1.483 & \\
\hline \multirow{3}{*}{$\mathrm{CoSe}_{2-\mathrm{x}}-\mathrm{Pt}$} & $1 \mathrm{M} \mathrm{KOH}$ & 1.485 & \\
\hline & & & Adv. Mater., 2019, 31, 1805581. \\
\hline & $0.1 \mathrm{M} \mathrm{KOH}$ & 1.544 & \\
\hline $\mathrm{CoFe} \mathrm{LDH} / \mathrm{Co}_{3} \mathrm{O}_{4}(6: 4)$ & $1 \mathrm{M} \mathrm{KOH}$ & 1.52 & ChemSusChem, 2019, 12, 4442-4451. \\
\hline $\mathrm{Ir} @ \mathrm{Co}$ & $1 \mathrm{M} \mathrm{KOH}$ & 1.503 & J. Mater. Chem. A, 2019, 7, 8376-8383. \\
\hline $\mathrm{Co}_{3} \mathrm{O}_{4} / \mathrm{NC}-350$ & $1 \mathrm{M} \mathrm{KOH}$ & 1.528 & Small, 2019, 1904260. \\
\hline $\mathrm{Co}_{3} \mathrm{O}_{4} \mathrm{HPNSs}-400$ & $1 \mathrm{M} \mathrm{KOH}$ & 1.538 & J. Mater. Chem. A, 2019, 7, 9059-9067. \\
\hline $\mathrm{Ru}-\mathrm{RuP}_{\mathrm{x}}-\mathrm{Co}_{\mathrm{x}} \mathrm{P}$ & $0.1 \mathrm{M} \mathrm{KOH}$ & 1.521 & Nano Energy, 2018, 53, 270-276. \\
\hline \multirow[t]{3}{*}{$\mathrm{Ni}_{3} \mathrm{Co}_{3} @ \mathrm{Ru} \mathrm{HNS}$} & $0.1 \mathrm{M} \mathrm{KOH}$ & 1.530 & \\
\hline & & & Small, 2018, 14, 1702353. \\
\hline & $1 \mathrm{M} \mathrm{KOH}$ & 1.502 & \\
\hline $\mathrm{Co}_{3} \mathrm{O}_{4} / \mathrm{HNCP}-40$ & $1 \mathrm{M} \mathrm{KOH}$ & 1.563 & ACS Catal., 2018, 8, 7879-7888. \\
\hline $\mathrm{NiCo}_{2} \mathrm{P}_{\mathrm{x}} / \mathrm{CNTs}$ & $1 \mathrm{M} \mathrm{KOH}$ & 1.514 & J. Mater. Chem. A, 2018, 6, 7420 . \\
\hline $\mathrm{Pd} @ \mathrm{PdO}-\mathrm{Co}_{3} \mathrm{O}_{4}$ & $1 \mathrm{M} \mathrm{KOH}$ & 1.54 & Adv. Energy Mater., 2018, 8, 1702734 \\
\hline $\mathrm{Co}_{3} \mathrm{O}_{4} / \mathrm{Co}-\mathrm{Fe}$ oxide & $1 \mathrm{M} \mathrm{KOH}$ & 1.527 & Adv. Mater., 2018, 30, 1801211. \\
\hline DSNBs & & & \\
\hline
\end{tabular}


Table S3. Summary of the state-of-the-art bifunctional oxygen electrocatalysts for ORR/OER in $0.1 \mathrm{M} \mathrm{KOH}$.

\begin{tabular}{|c|c|c|c|c|}
\hline Catalyst & $\begin{array}{l}E_{j=10}(O E R) \\
(V \text { vs RHE) }\end{array}$ & $\begin{array}{r}E_{1 / 2}(\text { ORR }) \\
(\mathrm{V} \text { vs RHE) }\end{array}$ & $\Delta \mathbf{E}(\mathbf{V})$ & References \\
\hline CoRu-O/A@HNC-2 & 1.483 & 0.821 & 0.662 & This work \\
\hline $\mathrm{CoSe}_{2-\mathrm{x}}-\mathrm{Pt}$ & 1.544 & 0.83 & 0.714 & $\begin{array}{l}\text { Adv. Mater., 2019, 31, } \\
1805581 .\end{array}$ \\
\hline PdCo-300 & 1.58 & 0.82 & 0.76 & $\begin{array}{l}\text { Appl. Catal. B-Environ., } \\
\text { 2019, 243, 175-182. }\end{array}$ \\
\hline $\mathrm{Co}_{9} \mathrm{~S}_{8} @ \mathrm{TDC}-900$ & 1.56 & 0.78 & 0.78 & $\begin{array}{l}\text { J. Mater. Chem. A, 2019, } 7 \text {, } \\
\text { 7389-7395 }\end{array}$ \\
\hline $\mathrm{CoDNi}-\mathrm{N} / \mathrm{C}$ & 1.59 & 0.86 & 0.73 & $\begin{array}{l}\text { Appl. Catal. B-Environ., } \\
\text { 2019, 240: 112-121. }\end{array}$ \\
\hline $\mathrm{Fe}_{3} \mathrm{C}-\mathrm{Co} / \mathrm{NC}$ & 1.601 & 0.885 & 0.72 & $\begin{array}{l}\text { Adv. Funct. Mater., 2019, } \\
\text { 29, } 1901949 .\end{array}$ \\
\hline $\mathrm{CoS}_{\mathrm{x}} / \mathrm{Co}-\mathrm{NC}-800$ & 1.54 & 0.80 & 0.74 & $\begin{array}{l}\text { Adv. Funct. Mater., 2019, } \\
\text { 29, } 1904481 .\end{array}$ \\
\hline NC@Co-NGC DSNC & 1.64 & 0.82 & 0.82 & $\begin{array}{l}\text { Adv. Mater., 2017, 29, } \\
1700874 .\end{array}$ \\
\hline $\mathrm{CoFe}_{2-\mathrm{x}} \mathrm{Zr}_{\mathrm{x}} \mathrm{O}_{4} / \mathrm{N}-\mathrm{rGO}$ & 1.57 & 0.73 & 0.84 & ACS Catal., 2018, 8, 3715. \\
\hline $\mathrm{Co}_{9} \mathrm{~S}_{8} @ \mathrm{TDC}-900$ & 1.56 & 0.78 & 0.78 & $\begin{array}{l}\text { J. Mater. Chem. A, 2019, } 7 \text {, } \\
\text { 7389-7395. }\end{array}$ \\
\hline $\begin{array}{l}\text { 2D Mesoporous FeCo- } \\
\mathrm{N}_{\mathrm{x}} \text {-Carbon }\end{array}$ & 1.67 & 0.886 & 0.78 & $\begin{array}{l}\text { Angew. Chem. Int. Edit., } \\
\text { 2018, 57, 1856-62. }\end{array}$ \\
\hline $\mathrm{CoIn}_{2} \mathrm{~S}_{4} / \mathrm{S}-\mathrm{rGO}$ & 1.6 & 0.83 & 0.77 & $\begin{array}{l}\text { Adv. Energy Mater., 2018, } \\
1802263 .\end{array}$ \\
\hline $\mathrm{CoS}_{\mathrm{x}} @ \mathrm{PCN} / \mathrm{rGO}$ & 1.57 & 0.78 & 0.79 & $\begin{array}{l}\text { Adv. Energy Mater., 2018, } \\
8,1701642 .\end{array}$ \\
\hline
\end{tabular}


Table S4. Summary of the state of the art bifunctional electrocatalysts for water splitting.in $1 \mathrm{M} \mathrm{KOH}$

\begin{tabular}{|c|c|c|c|c|}
\hline \multirow[t]{2}{*}{ Catalysts } & \multicolumn{2}{|c|}{$\begin{array}{c}\text { Overpotential } \\
\text { at } 10 \mathrm{~mA} \mathrm{~cm}-2(\mathrm{mV})\end{array}$} & \multirow{2}{*}{$\begin{array}{l}\text { water splitting } \\
\text { cell Voltage at } 10 \\
\qquad \mathrm{~mA} \mathrm{~cm}-\mathbf{~}(\mathrm{V})\end{array}$} & \multirow[t]{2}{*}{ References } \\
\hline & OER & HER & & \\
\hline CoRu-O/A@HNC-2 & 234 & 85 & 1.558 & This work \\
\hline $\mathrm{Co}_{1}-\mathrm{Fe}_{1}-\mathrm{B}-\mathrm{P}$ & 225 & 173 & 1.68 & $\begin{array}{l}\text { Nanoscale, 2019, 11, } \\
7506-7512\end{array}$ \\
\hline Co/CNFs (1000) & 320 & 190 & 1.69 & $\begin{array}{l}\text { Adv. Mater., 2019, 31, } \\
1808043\end{array}$ \\
\hline $\mathrm{Ni} / \mathrm{Mo}_{2} \mathrm{C}(1: 2)-\mathrm{NCNFs}$ & 288 & 143 & 1.64 & $\begin{array}{l}\text { Adv. Energy Mater., } \\
\text { 2019, 9, } 1803185\end{array}$ \\
\hline Co@N-CNTF-2 & 350 & 220 & $1.71 \mathrm{~V}$ & $\begin{array}{l}\text { J. Mater. Chem. A, } \\
\text { 2019, 7, 3664-3672 }\end{array}$ \\
\hline $\mathrm{CoP} / \mathrm{NCNHP}$ & 310 & 115 & 1.64 & $\begin{array}{l}\text { J. Am. Chem. Soc., } \\
\text { 2018, 140, 2610-2618 }\end{array}$ \\
\hline $\mathrm{Ni}-\mathrm{Co}-\mathrm{P} \mathrm{HNBs}$ & 270 & 107 & 1.62 & $\begin{array}{l}\text { Energy Environ. Sci., } \\
\text { 2018, 11, 872-880 }\end{array}$ \\
\hline $\mathrm{NCMC}$ & 290 & 169.5 & 1.63 & $\begin{array}{l}\text { Chem. Commun., } \\
\text { 2019, 55, 6515-6518 }\end{array}$ \\
\hline $\mathrm{Co}_{3} \mathrm{~S}_{4} / \mathrm{EC}-\mathrm{MOF}$ & 226 & 84 & 1.55 & $\begin{array}{l}\text { Adv. Mater., 2019, 31, } \\
1806672\end{array}$ \\
\hline $\mathrm{Ru}_{2} \mathrm{Ni}_{2} \mathrm{SNs} / \mathrm{C}$ & 310 & 40 & 1.58 & $\begin{array}{l}\text { Nano Energy, 2018, } \\
\text { 47, 1-7. }\end{array}$ \\
\hline $\mathrm{Co}_{3} \mathrm{O}_{4} @ \mathrm{Co}-\mathrm{MOF}-12$ & 277 & 172 & 1.637 & $\begin{array}{l}\text { Chem. } \\
\text { 2019, } \\
10907\end{array}$ \\
\hline
\end{tabular}

